\title{
Giant Goos-Hänchen shifts and radiation-induced trapping of Helmholtz solitons at nonlinear interfaces
}

\author{
Julio Sánchez-Curto, ${ }^{1, *}$ Pedro Chamorro-Posada, ${ }^{1}$ and Graham S. McDonald ${ }^{2}$ \\ ${ }^{1}$ Departamento de Teoría de la Señal y Comunicaciones e Ingeniería Telemática, \\ Universidad de Valladolid, ETSI Telecomunicación, Paseo Belén 15, Valladolid 47011, Spain \\ ${ }^{2}$ Joule Physics Laboratory, Materials and Physics Research Centre, School of Computing, \\ Science and Engineering, University of Salford, Salford M5 4WT, United Kingdom \\ *Corresponding author: julsan@tel.uva.es
}

Compiled August 10, 2011

Giant Goos-Hänchen shifts and radiation-induced trapping are studied at the planar boundary separating two focusing Kerr media within the framework of Helmholtz theory. The analysis, valid for all angles of incidence, reveals that interfaces exhibiting linear external refraction can also accommodate both phenomena. Numerical evidence of these effects is provided, based on analytical predictions derived from a generalized Snell's law. (C) 2011 Optical Society of America

OCIS codes: $190.3270,190.6135$

The evolution of optical beams at nonlinear interfaces involves a large variety of nonlinear phenomena which can not be described by the classical linear laws of reflection and refraction. Two such effects are the giant Goos-Hänchen shift (GHS) [1] and the capture or trapping of solitons at a nonlinear interface. The GHS, initially reported for optical beams at planar boundaries between two different linear media, has been quantified both theoretically [2-4] and experimentally [5, 6] using different approaches and techniques. The magnification of this effect, denoted as the nonlinear or giant GHS, was demonstrated in seminal works on nonlinear interfaces at boundaries separating linear and Kerr-type media [7,8]. Two nonlinear media were also found to accommodate giant GHS, as was shown in the context of particle-like theory for Kerr [9] and quadratic [10] media. In experiments, the giant GHS has been measured for linear-Kerr defocusing [11], linear-photorefractive [12] media and, more recently, for nematicons [13].

Nonlinear interfaces can also trap solitons impinging on the planar boundary at certain angles of incidence, so that they travel along the interface preserving their shape as localized nonlinear surface waves (NSW's). Since the works of Akhmediev [14], several studies have investigated excitation and stability of NSW's for a great variety of materials: two focusing Kerr [9,15], saturableKerr [16], diffusive-Kerr [17, 18], quadratic [10], defocusing Kerr [19] or thermal [20], and single or multilayered [21] configurations. Unlike these works, where radiative effects at the interface were negligible, radiation losses can play a central role in soliton trapping, and its subsequent propagation along the interface as a NSW [22]. Radiation-induced trapping has been reported for Kerr media embedded in nonlinear waveguides [23] and in structures with a periodic transverse modulation of the refractive index [24].

Most theoretical works devoted to either nonlinear GHS or radiation-induced trapping have been based on the Nonlinear Schrödinger (NLS) equation, thus restricting their validity to vanishingly small angles of incidence. Under such conditions, interfaces exhibiting linear internal refraction have traditionally been proposed for accommodating such effects. In this Letter, however, we show that both phenomena can also take place at interfaces with linear external refraction, whose inherent nonparaxial character can be properly captured within the context of an appropriate framework. Helmholtz theory $[25,26]$ overcomes the angular limitations of paraxial analyses, thus allowing study the evolution of broad beams (when compared to the wavelength) propagating at arbitrary angles. The theory is also valid for nonlinear interfaces [27,28], and uncovers numerical evidence of external GHS and radiation-induced trapping.

Figure 1 (a) illustrates the giant GHS $\zeta_{0}$ experienced by a Helmholtz bright soliton incident at angle $\theta_{i}$ on an interface separating two focusing Kerr media. The total refractive index of medium $i$ is $n=n_{0 i}+\alpha_{i} I, n_{0 i}$ is the linear refractive index, $\alpha_{i}>0$ the Kerr coefficient and $I$ the optical intensity. Soliton evolutions in Fig. 1 are obtained as solutions of the Nonlinear Helmholtz (NLH) equation $[25,29]$

$$
\begin{array}{r}
\kappa \frac{\partial^{2} u}{\partial \zeta^{2}}+j \frac{\partial u}{\partial \zeta}+\frac{1}{2} \frac{\partial^{2} u}{\partial \xi^{2}}+|u|^{2} u= \\
{\left[\frac{\Delta}{4 \kappa}+(1-\alpha)|u|^{2}\right] H(\xi) u}
\end{array}
$$

which has allowed us to quantify bright soliton reflection and refraction at interfaces separating two Kerr media $[27,28]$. Its derivation from the scalar Helmholtz equation is detailed in [28]. Here, $u$ is the complex envelope of a forward propagating beam associated with a TE optical field, $\xi=2^{1 / 2} x / w_{0}$ and $\zeta=z / L_{D}$ are the normalized transverse and longitudinal coordinates, respectively, and $w_{0}$ is the waist of a reference Gaussian beam with diffraction length $L_{D}=k w_{0}^{2} / 2 . H(\xi)$ is the Heavi- 
side function that represents the planar boundary, while interface parameters $\Delta=1-n_{02}^{2} / n_{01}^{2}$ and $\alpha=\alpha_{2} / \alpha_{1}$ account for the linear and nonlinear refractive index mismatch, respectively. $\kappa=1 / k^{2} w_{0}^{2}$ is a nonparaxiality parameter and $k=2 \pi / \lambda$ is the wavenumber in vacuum.

The GHS is linked to the existence of the critical angle for reflection, which within the Helmholtz framework for interfaces separating two focusing Kerr media is [27]

$$
\tan \theta_{c}=\left(\frac{\Delta+2 \kappa \eta_{0}^{2}(1-\alpha)}{1-\Delta+2 \kappa \eta_{0}^{2} \alpha}\right)^{1 / 2}
$$

where $\eta_{0}$ is the amplitude of the incident soliton. Predicitons of Eq. (2) in Fig. 1 (b) show that critical angles can exist at interfaces exhibiting both linear internal $(\Delta>0)$ and external $(\Delta<0)$ refraction. One thus suspect that external GHS may also occur at interfaces exhibiting linear external refraction. (a)
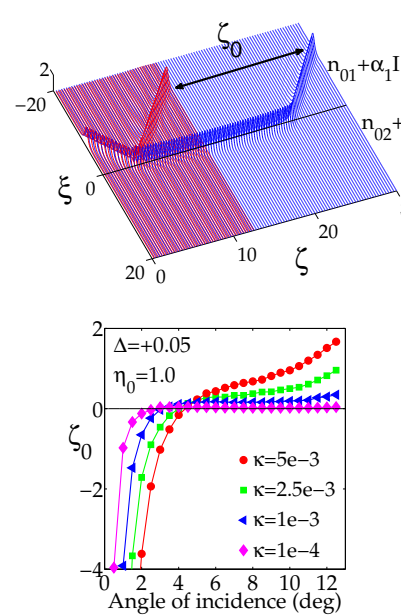

(c) (b)
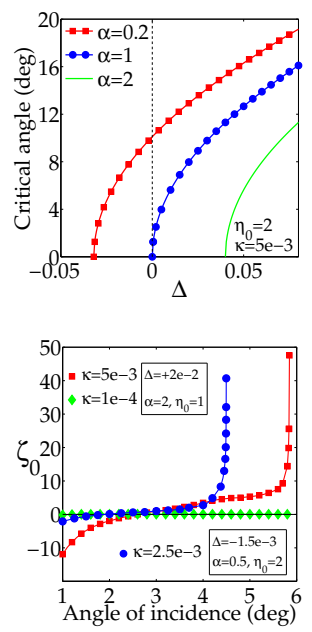

(d)
Fig. 1. (a) GHS for Helmholtz solitons. (b) Critical angle for diffrent nonlinear interfaces. (c) GHS found at linearstep interfaces depending on the beam size. (d) Giant GHS at nonlinear interfaces with $\Delta>0$ and $\Delta<0$.

We analyze first the GHS based on results obtained from full numerical integration of the NLH equation using a nonparaxial beam propagation method [30]. It is a difference-differential method which combines finite difference and spectral methods to compute the complex envelope of the evolving field. Figures 1 (c) and (d) show the magnitude of the linear and giant GHS obtained at linear-step $(\alpha=1)$ and nonlinear $(\alpha \neq 1)$ interfaces, respectively. Backward reflected waves at the interfaces are negligible in both cases. In order to highlight the dependence on beam size, the GHS for a fundamental soliton $\eta_{0}=1$ at a linear-step interface with $\Delta=0.05$ is calculated for four different beam sizes, containing approximately 5, 7, 10 and 32 wavelengths in the full width $2 \omega_{0}$ of a reference Gaussian beam $\left(\kappa=5 \times 10^{-3}\right.$, $\kappa=2.5 \times 10^{-3}, \kappa=10^{-3}$ and $\kappa=10^{-4}$, respectively).
The behaviour at linear-step interfaces depends strongly on beam size; the narrower the beam, the larger the displacement $[2,3]$. Negative values of $\zeta_{0}$ for small angles of incidence can be explained in terms of the particlelike model [9] developed within the framework of the paraxial theory which predicts that an incident soliton impinging the nonlinear interface at a very small angle of incidence will be repelled as the beam approaches the planar boundary. The role of $\kappa$ is also manifest in the analysis of the giant GHS shown in Fig. 1 (d). For a broad beam with $\kappa=10^{-4}$, the linear behaviour of the interface prevails as $\Delta \ll \kappa \eta_{0}^{2}$; no evidence of a giant GHS is revealed by the green (diamonds) line. This scenario changes completely when $\kappa=5 \times 10^{-3}$ and the critical angle predicted by Eq. (2) becomes $\theta_{c}=5.71^{\circ}$. The red (squares) line shows that a giant GHS is obtained as the soliton angle of incidence approaches $\theta_{c}$. We also report here, for the first time, external GHS occuring at interfaces with linear external refraction. This is shown by the blue (circles) line, where interface parameters and $\kappa$ have been chosen to give a different value of $\theta_{c}$. The small value of the angles in Fig.1 (d) is a result of the parameter set used in the simulations, considering that we work within the limits of a Helmholtz framework (broad beams when compared with the wavelength, i.e. $\kappa \ll 1$ ) and giant GHS arises at interfaces exhibiting a nonlinear behavior $2 \kappa \eta_{0}^{2}(1-\alpha) \sim \Delta$. For different parameters, the red curve of Fig.1 (d) can shift to much larger angles.

Unlike the usual giant GHS, external GHS demands a second medium with a lower Kerr nonlinearity, i.e. $\alpha<1$. Therefore, a bright soliton undergoing external GHS broadens on entering the new nonlinear media, as is shown in Fig. 2 (a). Just the opposite behaviour is found for the usual GHS at $\Delta>0$ interfaces in Fig. 2 (b), where the soliton narrows after entering a nonlinear medium with a larger Kerr nonlinearity. (a)

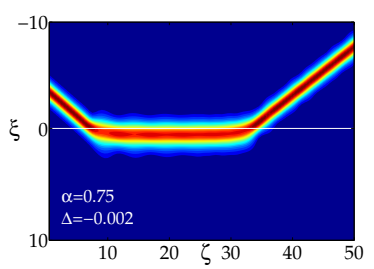

(b)

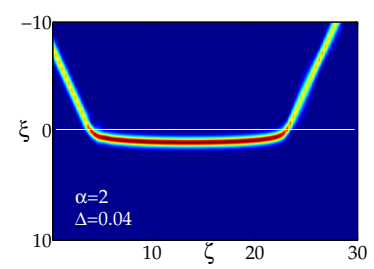

Fig. 2. (a) External and (b) usual Goos-Hänchen shift.

Radiation induced trapping for $\Delta<0$ is described by the same framework. Unlike previous paraxial studies where additional confinement mechanisms were needed to induce soliton trapping at the interface [23, 24], we show that, within the Helmholtz framework, this phenomenon can arise at interfaces separating two Kerr-type media without demanding any further conditions. This is illustrated in Fig. 3 (a), where soliton trapping induced by radiation losses arises as an equilibrium state resulting from two simultaneous counteracting effects. 
(a)
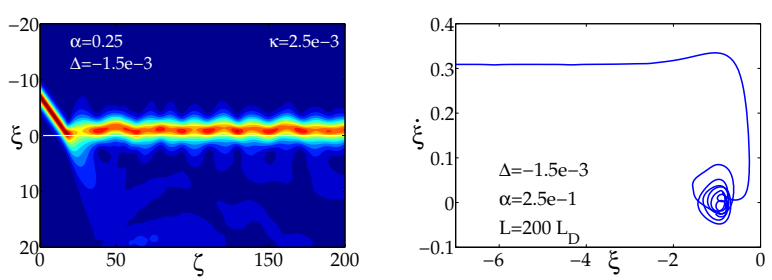

Fig. 3. (a) A Helmholtz soliton trapped by a nonlinear interface with $\Delta<0$. (b) Phase plane representation.

On one hand, the soliton tends to be reflected back into the first medium since interface parameters, $\kappa$ and angles of incidence, have been chosen in the vicinity of the critical angle [28]. On the other hand, as $\alpha<1$, the incoming soliton partially enters the new medium and undergoes spreading. Specular soliton reflection is thus avoided, and attempts to escape from the interface are counteracted by the attractive force of the $\Delta<0$ discontinuity. The effective soliton thus propagates for large distances $\left(\zeta_{0}=200\right)$ trapped by the interface. Such capture is illustrated in the phase plane of Fig. 3(b) representing the soliton position along the transverse coordinate $\xi$ and its velocity $\dot{\xi}$. Here, radiation losses are the result of a lower Kerr nonlinearity in the second medium. This makes a significant difference with previous approaches $[23,24]$, where radiation was induced by increasing the refractive index mismatch between adjacent media.

An accurate description of soliton trapping evidently also requires the full Helmholtz theory. If the numerical experiment shown in Fig. 3(a) is repeated with same parameter values but $\kappa$ is significatly reduced to $10^{-4}$, the generalized Snell's law reveals that critical angles are not allowed and the linear behaviour of the interface prevails. The incoming soliton thus enters the new medium undergoing external refraction.

Giant GHS can be interpreted as a behaviour close to the critical refraction at $\theta_{c}$ found within a geometrical optics description. Optical solitons are perfectly collimated, localized solutions with a well-defined propagation direction. They thus fit into an approximate description of an optical ray better than any linear solution with the same degree of localization in the transverse plane. As regards soliton trapping at the interface, radiation loss permits the adaptation of the refracted soliton to an effective critical value close to the expected angle of incidence.

This Letter has reported the formation of external GHS and radiation-induced soliton trapping at interfaces separting two focusing Kerr media, within the framework of Helmholtz theory. We have given numerical evidence of both phenomena taking place at interfaces exhibiting linear external refraction, and based predictions on analytical results of a generalized Snell's law.

\section{References}

1. F. Goos and H. Hänchen, Ann. Physik 1, 336 (1947).

2. T. Tamir and H.L. Bertoni, J. Opt. Soc. Am. 61, 1397 (1971).

3. B.R. Horowitz and T. Tamir, J. Opt. Soc. Am. 61, 586 (1971).

4. H.M. Lai, C.W. Kwok, Y.W. Loo and B.Y. Xu, Phys. Rev. E 62, 7330 (2000).

5. F. Bretenaker, A. Le Floch and L. Dutriaux, Phys. Rev. Lett. 68, 931 (1992).

6. E. Pfleghaar, A. Marseille and A. Weis, Phys. Rev. Lett. 70, 2281 (1993).

7. D. Marcuse, Appl. Optics 19, 3130 (1980).

8. W.J. Tomlinson, J.P. Gordon, P.W. Smith and A.E. Kaplan, Appl. Optics 21, 2041 (1982).

9. A.B. Aceves, J.V. Moloney and A.C. Newell, Phys. Rev. A 39, 1809 (1989).

10. I.V. Shadrivov and A.A. Zharov, J. Opt. Soc. Am. B 19, 596 (2002).

11. O. Emile, T. Galstyan, A. Le Floch and F. Bretenaker, Phys. Rev. Lett. 75, 1511 (1995).

12. B.M. Jost, A-A.R. Al-Rashed and B.E.A. Saleh, Phys. Rev. Lett. 81, 2233 (1998).

13. M. Peccianti, G. Assanto, A. Dyadyusha and M. Kaczmarek, Opt. Lett. 32, 271 (2007).

14. N.N. Akhmediev, V.I. Korneev and Yu.V. Kuzmenko, Zh. Eksp. Teor. Fiz. 88, 107 (1985).

15. L.A. Nesterov, Opt. Spektrosk. 64, 1166 (1988).

16. P.J. Bradley and C. De Angelis, Opt. Commun. 130, 205 (1996).

17. P. Varatharajah, A.B. Aceves and J.V. Moloney, J. Opt. Soc. Am. B 7, 220 (1990).

18. D.R. Andersen, Phys. Rev. A 37, 189 (1988).

19. Y. Chen, Phys. Rev. A 45, 4974 (1992).

20. Y.V. Kartashov, F. Ye, V.A. Vysloukh and L. Torner, Opt. Lett. 32, 2260 (2007).

21. D. Mihalache, M. Bertolotti and C. Sibilia, Prog. Opt. 27, 229 (1989).

22. Yu.S. Kivshar, A.M. Kosevich and O.A. Chubykalo, Phys. Rev. A 41, 1677 (1990).

23. Yu.S. Kivshar and M.L. Quiroga-Teixeiro, Phys. Rev. A 48, 4750 (1993).

24. Y.V. Kartashov, A.S. Zelenina, L. Torner and V.A. Vysloukh, Opt. Lett. 29, 766 (2004).

25. P. Chamorro-Posada, G.S. McDonald and G.H.C. New, J. Mod. Opt. 45, 1111 (1998).

26. P. Chamorro-Posada, G.S. McDonald and G.H.C. New, J. Mod. Opt. 47, 1877 (2000).

27. J. Sánchez-Curto, P. Chamorro-Posada and G.S. McDonald, Opt. Lett. 32, 1126 (2007).

28. J. Sánchez-Curto, P. Chamorro-Posada and G.S. McDonald, J. Opt. A: Pure Appl. Opt. 11, 054015 (2009).

29. G. Fibich, Phys. Rev. Lett. 76, 4356 (1996).

30. P. Chamorro-Posada, G.S. McDonald and G.H.C. New, Opt. Commun. 192, 1 (2001). 


\section{Informational Fourth Page}

In this section, please provide full versions of citations to assist reviewers and editors (OL publishes a short form of citations) or any other information that would aid the peer-review process.

\section{References}

1. F. Goos and H. Hänchen, "Ein neuer und fundamentaler Versuch zur Totalreflexion," Ann. Physik 1, 336346 (1947).

2. T. Tamir and H.L. Bertoni, "Lateral displacement of optical beams at multilayered and periodic structures," J. Opt. Soc. Am. 61, 1397-1413 (1971).

3. B.R. Horowitz and T. Tamir, "Lateral displacement of a light beam at a dielectric interface," J. Opt. Soc. Am. 61, 586-594 (1971).

4. H.M. Lai, C.W. Kwok, Y.W. Loo and B.Y. Xu, "Energyflux pattern in the Goos-Hänchen effect," Phys. Rev. E 62, 7330-7339 (2000).

5. F. Bretenaker, A. Le Floch and L. Dutriaux, "Direct measurement of the optical Goos-Hänchen effect in lasers," Phys. Rev. Lett. 68, 931-933 (1992).

6. E. Pfleghaar, A. Marseille and A. Weis, "Quantitative investigation of the effect of resonant absorbers on the Goos-Hänchen shift," Phys. Rev. Lett. 70, 2281-2284 (1993).

7. D. Marcuse, "Reflection of a Gaussian beam from a nonlinear interface," Appl. Optics 19, 3130-3139 (1980).

8. W.J. Tomlinson, J.P. Gordon, P.W. Smith and A.E. Kaplan, "Reflection of a Gaussian beam at a nonlinear interface," Appl. Optics 21, 2041-2051 (1982).

9. A.B. Aceves, J.V. Moloney and A.C. Newell, "Theory of light-beam propagation at nonlinear interfaces. I. Equivalent-particle theory for a single interface," Phys. Rev. A 39, 1809-1827 (1989).

10. I.V. Shadrivov and A.A. Zharov, "Dynamics of optical spatial solitons near the interface between two quadratically nonlinear media," J. Opt. Soc. Am. B 19, 596-602 (2002).

11. O. Emile, T. Galstyan, A. Le Floch and F. Bretenaker, "Measurement of the nonlinear Goos-Hänchen effect for Gaussian optical beams," Phys. Rev. Lett. 75, 15111513 (1995).

12. B.M. Jost, A-A.R. Al-Rashed and B.E.A. Saleh, "Observation of the Goos-Hänchen effect in a phase-conjugate mirror," Phys. Rev. Lett. 81, 2233-2235 (1998).

13. M. Peccianti, G. Assanto, A. Dyadyusha and M. Kaczmarek, "Nonlinear shift of spatial solitons at a graded dielectric interface," Opt. Lett. 32, 271-273 (2007).

14. N.N. Akhmediev, V.I. Korneev and Yu.V. Kuzmenko, "Excitation of nonlinear surface waves by Gaussian light beams," Zh. Eksp. Teor. Fiz. 88, 107-115 (1985).

15. L.A. Nesterov, "Total internal reflection and trapping of a plane soliton by the interface of nonlinear media," Opt. Spektrosk. 64, 1166-1168 (1988).

16. P.J. Bradley and C. De Angelis, "Soliton dynamics and surface waves at the interface between saturable nonlinear media," Opt. Commun. 130, 205-218 (1996).

17. P. Varatharajah, A.B. Aceves and J.V. Moloney, "Stationary nonlinear surface waves and their stability in diffusive Kerr-like nonlinear media," J. Opt. Soc. Am. B 7, 220-229 (1990).

18. D.R. Andersen, "Surface-wave excitation at the interface between diffusive Kerr-like nonlinear and linear media," Phys. Rev. A 37, 189-193 (1988).

19. Y. Chen, "Bright and dark surface waves at a nonlinear interface," Phys. Rev. A 45, 4974-4978 (1992).

20. Y.V. Kartashov, F. Ye, V.A. Vysloukh and L. Torner, "Surface waves in defocusing thermal media," Opt. Lett. 32, 2260-2262 (2007).

21. D. Mihalache, M. Bertolotti and C. Sibilia, "Nonlinearwave propagation in planar structures," Prog. Opt. 27, 229-313 (1989).

22. Yu.S. Kivshar, A.M. Kosevich and O.A. Chubykalo, "Radiative effects in the theory of beam propagation at nonlinear interfaces," Phys. Rev. A 41, 1677-1688 (1990).

23. Yu.S. Kivshar and M.L. Quiroga-Teixeiro, "Light-beam propagation at planar thin-film nonlinear waveguides," Phys. Rev. A 48, 4750-4757 (1993).

24. Y.V. Kartashov, A.S. Zelenina, L. Torner and V.A. Vysloukh, "Spatial soliton switching in quasi-continuous optical arrays," Opt. Lett. 29, 766-768 (2004).

25. P. Chamorro-Posada, G.S. McDonald and G.H.C. New, "Non-paraxial solitons," J. Mod. Opt. 45, 1111-1121 (1998).

26. P. Chamorro-Posada, G.S. McDonald and G.H.C. New, "Propagation properties of nonparaxial spatial solitons," J. Mod. Opt. 47, 1877-1886 (2000).

27. J. Sánchez-Curto, P. Chamorro-Posada and G.S. McDonald, "Helmholtz solitons at nonlinear interfaces," Opt. Lett. 32, 1126-1128 (2007).

28. J. Sánchez-Curto, P. Chamorro-Posada and G.S. McDonald, "Nonlinear interfaces: intrinsically nonparaxial regimes and effects," J. Opt. A: Pure Appl. Opt. 11, 054015 (2009).

29. G. Fibich, "Small beam nonparaxiality arrests selffocusing of optical beams," Phys. Rev. Lett. 76, 43564359 (1996).

30. P. Chamorro-Posada, G.S. McDonald and G.H.C. New, "Non-paraxial beam propagation methods," Opt. Commun. 192, 1-12 (2001). 